\title{
Non-Interpretative Analytic Techniques for Sicker Borderline Patients
}

\author{
Jaehak Yu and Jee Hyun Ha \\ Department of Psychiatry, Konkuk University Medical Center \& School of Medicine, Konkuk University, Seoul, Korea
}

Borderline personalities are known to be associated with sick and difficult patients in analysis. Sometimes usual interpretation in treating borderline personality patients is of no use due to the lack of acceptable attitude from these patients. Usually, non-interpretative analytic techniques such as containing (understanding of projective identification), holding, mirroring, scaffolding, blending, empathizing, and clarification of patients' status and affect are used before interpretations. These non-interpretative analytic techniques have been effective in treating sick borderline personality patients, especially when patients were not ready to accept interpretations. Various kinds of non-interpretative analytic techniques were reviewed in this study. Through this process, it was found that the effectiveness of these non-interpretative analytic techniques were not only prerequisites of therapeutic alliance for interpretation, but also independent therapeutic factors for the interaction between patients and analysts in treating sick patients with borderline personality.

Psychoanalysis 2017;28(3):51-57

KEY WORDS: Non-interpretative analytic techniques · Borderline personality $\cdot$ Containing $\cdot$ Projective identification $\cdot$ Holding Mirroring $\cdot$ Scaffolding $\cdot$ Blending $\cdot$ Empathizing $\cdot$ Clarification $\cdot$ Psychoanalysis.

Received: May 24, 2017 Revised: June 8, 2017 Accepted: June 8, 2017

Address for correspondence: Jaehak Yu, MD, PhD

Department of Psychiatry, Konkuk University Medical Center \& School of Medicine, Konkuk University, 120-1 Neungdong-ro, Gwangjin-gu, Seoul 05030, Korea

Tel: +82-2-2030-7566, Fax: +82-2-2030-7749, E-mail: drjaehakyu@yahoo.co.kr

\section{Introduction}

Borderline personalities have been regarded as one of the sicker and more difficult patients in analysis. It is because they show extreme impulsivity, difficulty in establishing object constancy, use of more primitive defenses such as splitting, and projective identification during analytic sessions. It is also because they bring resultant reactive negative countertransference feelings from patients' analysts.

What we are trying to say is not about the characteristics of borderline patients in this paper. What we want to discuss is how to facilitate usual interpretation in treating borderline patients. Sometimes, usual interpretation in treating borderline personality patients is of no use due to the patients' lack of acceptable attitude. Usually, non-interpretative analytic techniques such as container/contained or containing (understand-

This is an Open Access article distributed under the terms of the Creative Commons Attribution Non-Commercial License (http://creativecommons.org/licenses/by-nc/4.0) which permits unrestricted non-commercial use, distribution, and reproduction in any medium, provided the original work is properly cited.

- Part of the paper was presented on the day of 4th of May 2017 in the IPA Asia-Pacific Conference which was held in Regent Hotel in Taipei, Taiwan from May 4th 2017 to May 6th 2017. ing of projective identification), holding, mirroring, scaffolding, blending, empathizing, and clarification of patients' status and affect are used before interpretations.

There are many psychoanalytic papers that discuss a single non-interpretive analytic technique, such as holding and containing. However, there are only a few papers which examine several non-interpretative analytic techniques at the same time. The latter may be due to the fact that many analysts believed all the non-interpretative analytic techniques portray the same meaning even though different analysts described different non-interpretative analytic techniques or explained the same phenomenon with different points of view.

The characteristics that all non-interpretative psychoanalytic techniques such as containing through understanding of projective identification, holding, mirroring, scaffolding, blending, empathizing, and clarification of patient's status \& affect have in common are as follows: acceptance (Pedersen et al. 2014), openness (Pedersen et al. 2014), fine tuning, seeing through, no revenge against patient (Dowling 2016), no exclusion of patient, sharing with patient, and playing with patient.

However, we don't think it would be enough for us to know the common characteristics of all non-interpretative psychoanalytic techniques in treating sicker patients such as those with 
borderline personality disorder. Rather, we need to know the specific and various meanings of each non-interpretative psychoanalytic technique. We believe that the more we know each of these non-interpretative psychoanalytic techniques, the more chances we have to treat sicker patients. That is the reason why we try to figure out the differences among non-interpretative psychoanalytic techniques, especially those between containing and holding in this paper.

The study of non-interpretative psychoanalytic techniques, especially containing and holding, goes along the lines of recent studies on two-person psychology such as object relations theories, relational models, and intersubjectivist's models. This recent trend of emphasis on two-person psychology is associated with the fact that there are similarities between the psychoanalytic inter-relational process and early interaction between mother and baby. In other words, both containing and holding are critical to both the psychoanalytic inter-relational process and early interaction between mother and baby.

Emde (2005) mentioned five similarities between analytic inter-relational process and early interaction between mother and baby in detail. These five similarities are as follows.

First of all, we have to mention two-person psychology. Both the early mother-infant relationship and the analyst-sicker patient relationship are dual relationships and there is no single baby (patient) or mother (therapist) as Bowlby (1969) mentioned. Two-person psychology also means that there will be no interaction, and as a result, no change if there is no real participation from either the patient or analyst. Therefore, enactment is regarded as evidence of interaction between the patient and analyst. The implication here is that the enactment is ubiquitous and it is very important not to be afraid of being enacted on by the patient or enacting as an analyst. We would like to mention one more thing about two-person psychology because I think it is very important clinically. According to Emde (2005), special conditions are required for sharing to occur: a shared zone of understanding for both the patient and the analyst, a shared sense of openness about what can be expressed, a shared sense of safety, and a shared sense of restraint about what should not happen. Especially we think that a shared sense of restraint that actions should not happen in analytic consulting room makes a patient associate and bring unconscious materials more freely in the practice of psychoanalysis.

Second, we will bring up the importance of availability. One of the most important duties of analyst is being available whenever the patient is in need of the analyst. This is as important as the mother's availability when a baby is in need of his mother. Through this availability, basic trust, confidence, and object constancy are achieved. This availability becomes a presupposition of the therapeutic alliance. It is also very important for us as clinicians to know that availability becomes manifest through regulation and balance, which means that an analyst tries to escape extreme attitudes and maintain individual integrity.

Third, we will talk about affective mirroring and affective scaffolding. It is the same discussion as the one mentioned when I bring up the third and fourth points in introducing non-interpretative analytic techniques.

Fourth, we will talk about the empathetic mother and analyst. We would like to mention one thing here about the corrective empathic experience, which is different from Alexander (1950)'s corrective emotional experience. In corrective empathic experiences, the analyst is not a benevolent object who supplies a lacking part of a patient but a neutral object who understands and conveys to a patient his or her lacking part.

Fifth and last of all, proper interpretation for a patient and a child is eventually needed for permanent change of personality structure and individuation. In doing interpretation we have to be careful because all interpretation should be proper not only for the developmental level of the child and patient, but also for individual capability and personal competence of the child and patient. One aspect of neutrality is to interpret according to the patient's ability, not according to the analyst's hope or desire.

It is not true that one-person psychologists such as orthodox Freudians and ego psychologists disregard the importance of relationship between a patient and his analyst. They thought that this relationship factor is also important in psychoanalysis. It is understood that they thought a good relationship between a patient and his analyst is a prerequisite for a therapeutic alliance which will make the analyst's interpretation and the patient's insight more efficient. In other words, good therapeutic alliance increases effectiveness of interpretations. The followings are some of the many examples which emphasized the importance of therapeutic alliance: the unobjectionable positive transference and alliance (Freud 1965), corrective emotional experience (Alexander 1950), analytic situation (Stone 1973), working alliance (Greenson 1965), real relationship (Greenson and Wexler 1969), and passing the patient's test (Weiss 1988).

\section{Various Kinds of Non-Interpretative Analytic Techniques}

\section{Container/contained}

As you know, container/contained is Bion's concept. It is the extension to an interpersonal domain of (intra-psychic) projective identification. An infant/patient projects his own mental contents that he does not know well (especially affectladen mental contents) to his mother/analyst. Then the mother/ 
analyst contains (the term "container" comes from the mother/ analyst who contains actively and "contained" comes from the infant/patient who is contained passively) or accepts her infant's/patient's affect-laden mental contents. In the next step, the mother/analyst thinks thoroughly about what her infant/patient projects, and finds out the meanings of her infant's/patient's projections in her own mind. Then the mother/analyst shows the infant/patient her digested meanings of his projected mental contents. The infant/patient may identify or become aware of the digested meanings of his projected mental contents. Finally, the infant/patient begins to understand what he did not know before and this understanding is achieved through the mother's/analyst's process of reverie. Another thing to mention about Bion's container/contained concept is that through this process, the infant/patient learns how to think about and how to deal with experiences that he did not know before through internalization of the mother's/analyst's role of container (Bion 1965).

Ogden (2004) extended the meaning of containing. He mentioned that container/contained is not the object, nor what the object did, but how we deal with lived emotional experiences and what would happen if we did not deal with these lived emotional experiences. He explained that the integral part of container/contained is the process of turning what we did not know about the lived emotional experiences into what we do know about the lived emotional experiences of the past. He further explained that the process of container consists of unconscious dreaming, preconscious reverie, and conscious reflection, with unconscious dreaming being the most powerful and important. According to him, also, the process of contained is not fixed but constantly changing and expanding like a living organism through containing, which includes dreaming, thinking, remembering, and through interactions between mother/analyst and baby/patient. In the process, raw sense impression and unthought-like thought (beta elements) came to have meanings which were connected with important past experiences (alpha element). Conclusively, he mentioned that contained is composed of thoughts and feelings from lived emotional experiences of the past and that container consists of abilities such as thinking, dreaming, and remembering which make meaningful thoughts and feelings. Therefore, the aim of psychoanalysis is not the resolution of the patient's conflicts but the expansion of the patient's ability to think and dream of lived emotional experiences of which he did not know the meaning in the past (Ogden 2004).

Before going to the next non-interpretative intervention, I have to mention Klein's projective identification (Klein 1985) because the concept of container/contained stems from the concept of projective identification.

In the early days, projective identification was thought to be a primitive form of defense. It means that the infant/patient tears off or removes a bad part of his own mental content and projects it to his mother/analyst. Through this process, the infant/patient sees the bad part in his mother/ therapist and does not feel that he is bad because his bad part is not inside himself but inside his mother/analyst. In the early stages, the aims of projective identification for the infant/patient are thought to destroy his mother/analyst and to control his mother/analyst, but gradually the analysts come to think that one of most important aims of infant's/patient's projective identification is communicating unconsciously with his mother/analyst. Clinically, it is important for the mother/analyst to recognize her emotions being stirred up when the infant/patient is unconsciously using projective identification. Both the infant/patient and mother/analyst will not know about the infant's/patient's use of projective identification if mother/ analyst does not know that the infant/patient is using projective identification.

Nowadays, the meaning of projective identification is thought to be similar to the concept of container/contained. That means the infant/patient uses projective identification to solve his own problems. It is thought that the infant/patient projects to his mother/analyst if he does not understand his intense emotional experiences. Then, the mother/analyst accepts and transforms these intense emotional experiences to tolerable forms of those experiences. The final step of projective identification is the infant's/patient's identification of the mother's/analyst's tolerable forms of experiences, which are quite emotionally controllable.

\section{Holding}

Second of all, I will go over Winnicott's holding function of the mother/analyst (Winnicott 1960). As a pediatrician, Winnicott thought that techniques of the mother's physical holding, bathing, and feeding constituted everything she did for her baby. Therefore, physical holding gives the baby the first idea of a mother and establishes for the baby a place where he can live securely. Also, physical holding is based on the baby-mother bond. As an analyst, Winnicott thinks that correct and welltimed interpretation gives off a sense of physical holding.

Winnicott further mentions that proper emotional holding that the mother/analyst supplies satisfies the infant's/patient's affective needs. Through emotional holding, a good enough mother/analyst gives the baby/patient natural skill and object constancy. A holding mother is different from an object mother, who supplies complete satisfaction for baby's wishes from the beginning. A holding environmental mother always stays in the baby's environment and tells her baby his status constantly. That means that the holding mother cannot satisfy the baby's wishes completely and is only able to tell the baby whether he is satisfied or frustrated with something. 
Ogden (2004) also mentioned holding. He thought of holding as an ontological concept, in which a baby becomes aware of his existence (his being). It is through the process in which his mother's existence is shown to him by his mother. I think he emphasized the importance of the function of the mother in making her baby become aware of his existence. He also mentioned different kinds of holding such as being in the infant's time, the gathering of bits, internalization of the holding environment and depressive position holding as parts of a developmental process.

Slochower (1991) also mentions various kinds of holding. First, he mentions that the analyst supplies a regressive part of holding if the patient shows ego deficit for the time being. Second, he mentions a holding environmental mother, in which case the mother/analyst does not supply gratification such as feeding and bathing. Instead, the mother/analyst performs the function of an environmental mother/analyst, such as providing neutrality and object constancy. Third, he equates the survival of the analyst with holding. It means that the analyst maintains functions of analyst even if there are aggressive and destructive wishes from the patient, including when the analyst's countertransference keeps the treatment uninterrupted. Fourth, he discusses holding of the patient's acting out, in which the analyst can continue treatment for the patient

The analytic situation also provides a holding environment through regularity of visits, rituals of coming and going, underlying empathy, steadiness of therapist's voice, supportive continuity of objects, and spaces and textures of the analytic room.

\section{Affective mirroring}

Third, I will discuss affective mirroring. Like the concepts of container/contained and holding, the concept of affective mirroring exhibits characteristics of both the early developmental process and the analytic process. The mother/analyst pays attention to the emotional status of the infant/patient, and the infant's/patient's emotional status is reflected by the mother's/analyst's mind as if with a mirror. Then the mother/ analyst comes to understand the infant's/patient's affective status by monitoring her (the mother/analyst) own emotional status. This concept of emotional mirroring is quite different from Freud's metaphor of the mirror, in which the mother/analyst does not feel or show her own emotional status to the infant/patient at all.

According to Kohut (1984), there are two lines of development which is composed of development of object libido and narcissistic libido. The narcissistic libido develops with the process called transmuting internalization which needs primary caregiver's mirroring and optimal frustration. Kohut said mirroring enhances the development of grandiose-self through the process of the gleam in the mother's eye mirroring the child's narcissistic exhibitionistic unfolding. That means the mother supplies the child a feeling of omnipotence and affirms the child's self-esteem by mirroring.

Gergely and Watson (1996) also extend meanings of mirroring. They show that through parental affective mirroring the infant first achieves the sensitization function; the infant becomes aware of his own internal status and emotions as if biofeedback were used. Second, the infant also achieves the representation building function in which the infant forms secondary representation through his connection with reflective displays of primary emotions. Third, the infant also achieves the state-regulation function which lessens the need for expression of negative emotion through soothing interaction with his parent. Finally, the infant achieves the communicative and mentalizing function by learning the meanings of his own emotions and by transmitting a new pretend mode between him, the infant, and his parent.

\section{Affective scaffolding}

Fourth, I will mention the provision of affective scaffolding. Literally, scaffolding consists of poles and boards made into a framework similar to that used by construction workers. The mother's/analyst's provision of affective scaffolding gives the infant/patient an ability to understand others' emotions. By providing affective scaffolding, the mother/analyst proves that she has the ability to soothe and comfort her infant/patient. The mother's/analyst's provision of affective scaffolding is especially necessary when the infant's/patient's ability to tolerate intense emotions is inadequate. The mother/analyst has to know about her infant's/patient's ability to cope with emotions and provide the proper amount of emotions which are digestible to the infant/patient. That means that one of the mother's/analyst's roles is to protect her infant/patient against overstimulation (Shengold 1989).

\section{Blending}

Fifth, I will talk about blending. Literally, blending is to mix different kinds of things together. Blending emotions which are projected to the mother/analyst by the infant/patient is a very important process because through this process, the mother/analyst may find out digestible or controlled meanings of emotions especially when emotions are too negative or too intense.

Another meaning of blending emotions is thought to be the mother's/analyst's fine tuning toward and sorting out of emotions that the infant/patient projects to his mother/analyst. Through this process of blending, the mother/analyst comes to know what the infant/patient is trying to convey to her. 


\section{Empathy}

Sixth, I will talk about empathy as a non-interpretative analytic intervention. As Sugarman (2008) suggested, empathy does not seem to be a goal in psychoanalytic treatment, but it seems to be one of the many methods which brings about the therapeutic process in psychoanalytic treatment. Therefore, empathic attitude is something that the mother/analyst can try from the first session, but creating actual empathy for the infant/patient is a process which requires time and numerous interactions between the infant/patient and the mother/analyst. It is important for us as analysts to recognize what kinds of feelings the patient has (emphasizes) subjectively during treatment sessions. However, it is also important to understand objectively or to observe what goes on between the patient and analyst during treatment sessions. To objectively observe what goes on during the session, the analyst has to be less narcissistic and more neutral in her stance. We agree with Raphling (1991) who mentioned that to empathize is not the analyst's doing, but the patient's permitting of the analyst to do so through their interaction during treatment sessions.

\section{Clarification}

Seventh, I will talk about clarification. According to Gabbard (2005), clarification is one of the most frequently used non-interpretative analytic interventions and also represents one of the key elements of the psychoanalytic process (clarification-interpretation-working through). Through clarification, the analyst reformulates or pulls together what the patient has told the analyst. The analyst also tries to help the patient talk more coherently through clarification if the patient has difficulty in verbally expressing what he wants to convey. Clarification is different from confrontation in that the analyst does not point out defense mechanisms such as denial and minimization.

We think, however, it is more important for us to know the importance of clarification as a way to facilitate verbal expression and as a way to clarify or to let the patient know his current status, especially his current status of emotions. Sometimes clarification of the patient's emotions is also thought to be more valuable than interpretation because it gives the patient a chance to stay in the emotional state longer instead of being forcibly taken out of the emotional state through cognitive intervention of interpretation. In other words, a clarification of the patient's emotion such as "You seem to be very angry ..." is more helpful for the patient to associate freely rather than an interpretation of the patient's emotion such as "You seem to be very angry with me because you thought that I did not understand you at all $\cdots$." This is because the analyst's cognitive explanation of the patient's anger is sometimes misunderstood by the patient as a command to stop being angry with the analyst since the analyst knows the reason of the patient's anger.

\section{Non Interpretative Analytic Techniques for Improvement of Interaction as a Therapeutic Factor}

Many analysts in the past seem to have thought that these non-interpretative analytic techniques are prerequisites to achieve therapeutic alliance, which makes for more effective interpretation. Many analysts today, however, seem to think that these non-interpretative analytic techniques are an independent therapeutic factor which enhances the interaction between the patient and the analyst especially in treating sicker patients such as borderline personality disorder patients.

In his paper about the psychoanalytic process, Abrams (1990) mentioned two psychoanalytic processes or therapeutic factors. One is insight which is brought by the analyst's interpretation or his own self-analysis, which is an extension of the patient's ability to integrate his intra-psychic process. The other is the therapeutic effect from the interpersonal aspect between the analyst and patient. Abrams (1990) also mentioned that this interpersonal therapeutic effect and other object relations approaches are not usually regarded real therapeutic factors because these are developmental and quite different from psychoanalytic "intra-psychic" changes. However, we agree with his conclusion that analytic processes or therapeutic factors have two aspects: intra-psychic insight and interpersonal interaction between the patient and the analyst. In short, there are two psychoanalytic processes, which are integration and development.

Many analysts today think that there are surely relational therapeutic actions. The evidence of relational therapeutic actions is associated with the following: first, the cognitive aspect of interpretation is less important than the emotional aspect of interpretation. Second, there are almost no analysts who emphasize the importance of the revival of repressed memory and the importance of reconstruction in psychoanalysis. That means transference is more important than reconstruction. Third, for treatment to be effective, the patient has to understand the transference phenomena and be understood by the analyst in the situation at hand. Fourth, a therapeutic relationship means that the patient comes to an understanding in the interaction with the analyst.

\section{Discussion}

Many analysts use the concepts container/contained and holding interchangeably. It is understandable that both container/contained and holding include communicative process 
between mother/analyst and infant/patient. It is also understandable that through the mother/analyst's empathic understanding in the process of holding and container/contained, the infant's/patient's frustration can be relieved enormously. Also in the process of holding and contained/contained, a well-adapted mother/analyst can give an infant/patient what he wants through accurate recognition of the infant's/patient's needs.

In discussing the differences between the concepts container/contained and holding, Pedersen et al. (2014) thought holding is a wider concept than container/contained. It is because holding encompasses all mother-child relationships and containing only includes the operationalization of the mother's detoxifying or regulating her child's unbearable internal state, which is similar to Gergely and Watson (1996)'s affect regulation.

However, we think the concept of container/contained means that the mother/analyst permits a more unconsciously driven interaction between herself and her infant/patient than in holding. That is, in holding, the mother/analyst points out the infant's/patient's status on a more conscious level. On the other hand, in containing, the mother/analyst permits her infant's/patient's unconscious projection and projective identification; as a result, the mother/analyst comes to a more thorough understanding through resultant unconscious interaction between herself and her infant/patient.

Then our next question is, what is the difference between Freud's free floating attention or evenly suspended attention and containing by the mother/analyst? In Freud's era, using the analyst's own unconscious contents in understanding patient's unconscious contents was not recommended. Therefore, it did not seem to be advisable to use the analyst's own feelings, memory, and thoughts about the patient in doing free floating attention. Free floating attention was just for neutrality through more attention toward the patient.

Sandler (1992), however, made the term free floating responsiveness, which showed dynamic to-and-fro interaction between patient and analyst. I think this free floating responsiveness makes us to think about Freud's free floating attention again recently.

Then, I think there is actually no difference between Freud's free floating attention and Bion's containing when these techniques are used clinically. In containing, the analyst seems to use all kinds of her own feelings, memory, and thoughts about the patient to understand the patient and to enhance interaction between herself and the patient.

Then we may come to another question: whether or not all that we feel and think during sessions with patients come from the patients' projection or projective identification. Since Segal (1973) mentioned the usefulness of countertransference, almost all analysts today understand and accept that the analyst's countertransference can be used in understanding the patient's unconscious mind. How far can we use our own countertransference? As far as I know, very important point of accepting and understanding the patient's projective identification is the point of our emotions being stirred up, which is quite different from our ordinary emotional status. However, as far as I understand Bion's concept of containing, we can use not only the moment that our emotions are stirred up, but also any slight or minimal changes in our emotions in understanding the patient's unconscious mind. I am not sure whether our ordinary emotions can be thought to be from our patients' mind rather than from our own minds. These ordinary emotions of ours can be thought of as our own countertransference or counter-identification.

Even today, the relational, intersubjectivity model recommends analysts use self-disclosure to enhance the interaction between analyst and patient. According to the model, through this enhancement of interaction, analysts will have more chances to understand the patient. However, I am not sure how far I can use my own feelings, memory, and thoughts in understanding patients and how far I can expose myself to the patient as a trainee in ego psychology.

\section{Conclusion}

Current analysts found that these non-interpretative analytic techniques were effective in treating sicker borderline personality patients especially when the patients were not ready to accept interpretations. Particularly, the concept of container/contained is important in containing or accepting what the patient is projecting to the analyst. Also, the concept of holding includes the analyst's provision of neutrality and object constancy.

The authors think that these non-interpretative analytic techniques are not only prerequisites in the process of achieving therapeutic alliance for interpretation, but also independent therapeutic factors of interaction between patients and therapists in treating borderline personality patients. However, we do not know how far we use our own feelings and thoughts during the sessions with the patients and how far we use selfdisclosure to enhance the interactions between analytic dyad when we use these non-interpretative analytic techniques such as containing and holding.

\section{Conflicts of Interest}

The authors have no financial conflicts of interest.

\section{REFERENCES}

Abrams S. The psychoanalytic process: the developmental and the integrative. Psychoanal Q 1990;59:650-677. 
Alexander F. Analysis of the therapeutic factors in psychoanalytic treatment. Psychoanal Q 1950;19:482-500.

Bion WR. Transformations: change from learning to growth. London: Heinemann; 1965.

Bowlby J. Attachment and loss: volume I: attachment. London: The Hogarth Press and the Institute of Psycho-Analysis;1969.

Dowling AS. Personal communication. 2016.

Emde RN. A developmental orientation for contemporary psychoanalysis. In: Gabbard G, Person E, Cooper A. Textbook of psychoanalysis. Washington, DC: American Psychiatric Publishing;2005. p.117-130.

Freud S. Remarks on the theory and practice of dream-interpretation. The standard edition of the complete psychological works of Sigmund Freud, Volume 19. Translated by Strachey J. London: Hogarth Press; 1965.

Gabbard GO. Psychodynamic psychiatry in clinical practice. 4th ed. Washington, DC: American Psychiatric Publishing;2005.

Gergely G, Watson JS. The social biofeedback theory of parental affectmirroring: the development of emotional self-awareness and selfcontrol in infancy. Int J Psychoanal 1996;77(Pt 6):1181-1212.

Greenson RR. The working alliance and the transference neurosis. Psychoanal Q 1965;34:155-181.

Greenson RR, Wexler M. The non-transference relationship in the psychoanalytic situation. Int J Psychoanal 1969;50:27-39.

Klein HS. A Kleinian point of view. J Child Psychother 1985;11:31-47.

Kohut H. How does analysis cure? Edited by Goldberg A. Chicago:
University of Chicago Press;1984.

Ogden TH. On holding and containing, being and dreaming. Int J Psychoanal 2004;85(Pt 6):1349-1364.

Pedersen SH, Poulsen S, Lunn S. Affect regulation: holding, containing and mirroring. Int J Psychoanal 2014;95:843-864.

Raphling DL. Understanding countertransference: from projective identification to empathy: by Michael J Tansey and Walter F Burke. Hillsdale, NJ: The Analytic Press, 1989. 222 pp. Psychoanal Q 1991;60:121-124.

Sandler J. Reflections on developments in the theory of psychoanalytic technique. Int J Psychoanal 1992;73(Pt 2):189-198.

Segal H. Introduction to the work of Melanie Klein. London: Hogarth Press; 1973.

Shengold L. Further thoughts about "nothing". Psychoanal Q 1989;58: 227-235.

Slochower J. Variations in the analytic holding environment. Int J Psychoanal 1991;72:709-718.

Stone L. On resistance to the psychoanalytic process: some thoughts on its nature and motivations. Psychoanal Contemp Sci 1973;2:42-73.

Sugarman A. Fantasizing as process, not fantasy as content: the importance of mental organization. Psychoanal Inq 2008;28:169-189.

Weiss J. Testing hypotheses about unconscious mental functioning. Int $\mathrm{J}$ Psychoanal 1988;69(Pt 1):87-95.

Winnicott DW. The theory of the parent-infant relationship. Int J Psychoanal 1960;41:585-595. 